\title{
Rapid quantification of tissue perfusion properties with a two-stage look-up table: a simulation study
}

\author{
BIN YANG ${ }^{*}$ AND WILLIAM MILLeR
}

Duquesne University, Department of Engineering, Pittsburgh, USA

*E-mail: yangb1@duq.edu

\begin{abstract}
Tissue perfusion properties reveal crucial information pertinent to clinical diagnosis and treatment. Multispectral spatial frequency domain imaging (SFDI) is an emerging imaging technique that has been widely used to quantify tissue perfusion properties. However, slow processing speed limits its usefulness in real-time imaging applications. In this study, we present a two-stage look-up table (LUT) approach that accurately and rapidly quantifies optical (absorption and reduced scattering maps) and perfusion (total hemoglobin and oxygen saturation maps) properties using stage-1 and stage-2 LUTs, respectively, based on reflectance images at $660 \mathrm{~nm}$ and $850 \mathrm{~nm}$. The two-stage LUT can be implemented on both CPU and GPU computing platforms. Quantifying tissue perfusion properties using the simulated diffuse reflectance images, we achieved a quantification speed of 266, 174, and 74 frames per second for three image sizes 512x512, 1024x1024, and 2048x2048 pixels, respectively. Quantification of tissue perfusion properties was highly accurate with only $3.5 \%$ and $2.5 \%$ error for total hemoglobin and oxygen saturation quantification, respectively. The two-stage LUT has the potential to be adopted in existing SFDI applications to enable real-time imaging capability of tissue hemodynamics.
\end{abstract}

Keywords: spatial frequency domain imaging, hemodynamics, perfusion properties, Monte Carlo simulation, look-up table

\section{Introduction}

Sufficient oxygen supply in tissue is critical to maintaining its normal physiological functions [1,2]. Local oxygen levels in tissue provide valuable insights into its health states, such as metabolic levels [3]. Oxygen is transported via binding to hemoglobin, the main protein of red blood cells, to different parts of the body, and is released to tissue through perfusion. Depending on the presence of binding oxygen molecules, hemoglobin can exist in two forms, oxygenated and deoxygenated hemoglobin, both of which exhibit distinct absorption properties in visible and near-infrared (NIR) ranges. Based on the differential absorption of hemoglobin, multispectral imaging was developed to quantify tissue perfusion properties, such as oxygen saturation[4]. However, the quantification accuracy was limited due to its inability to decouple tissue absorption from tissue scattering. Spatial frequency domain imaging (SFDI), on the other hand, independently evaluates tissue absorption and scattering properties using 2D structured illuminations [5-7]. Multispectral SDFI has been developed to map perfusion and hemodynamic properties of the tissue with an improved accuracy [8,9]. Such quantification requires imaging at multiple wavelengths (two wavelengths at least), typically in the red and near-infrared (NIR) range [10, 11].Typically, at each wavelength, a set of 3 phase-shifted patterns are projected to the tissue surface and the 
corresponding reflectance images are acquired to determine the tissue's optical and perfusion properties [6].

The overall process, however, is slow and limits the temporal resolution. In the past few years, significant efforts have been devoted to improving the imaging and quantification speed in SFDI imaging. Single snapshot SFDI reduced the required images from 3 to 1 [12-14], and look-up table (LUT) based quantification significantly improved the quantification speed of optical property of the tissue [15]. For perfusion property quantification, Beer-Lambert Law is the standard approach, which could be slow for large images. To improve the speed for perfusion property quantification, artificial intelligence was employed to train the predictive model based on a large set of training images $[16,17]$.

Despite recent advancements towards real-time mapping of tissue perfusion and hemodynamics, current methods are still limited by relatively slow processing speed, low image resolution, and the need for powerful computational hardware (e.g., multiGPU setup). This study aimed to develop a highly efficient LUT-based computational framework towards high-resolution real-time mapping of tissue perfusion and hemodynamics that can be implemented on cost-effective hardware platforms.

\section{Methods}

LUT techniques have been widely used in SFDI to determine optical properties without performing time-consuming fitting procedures [18]. However, LUT techniques were rarely used to quantify tissue perfusion properties in conjunction with SFDI imaging. In this study, we developed a highly efficient two-stage LUT technique consisting of two connected LUTs. The first and second stage LUTs rapidly quantify tissue optical and perfusion properties, respectively. Fig. 1 summarizes the overall workflow of the two-stage LUT technique.

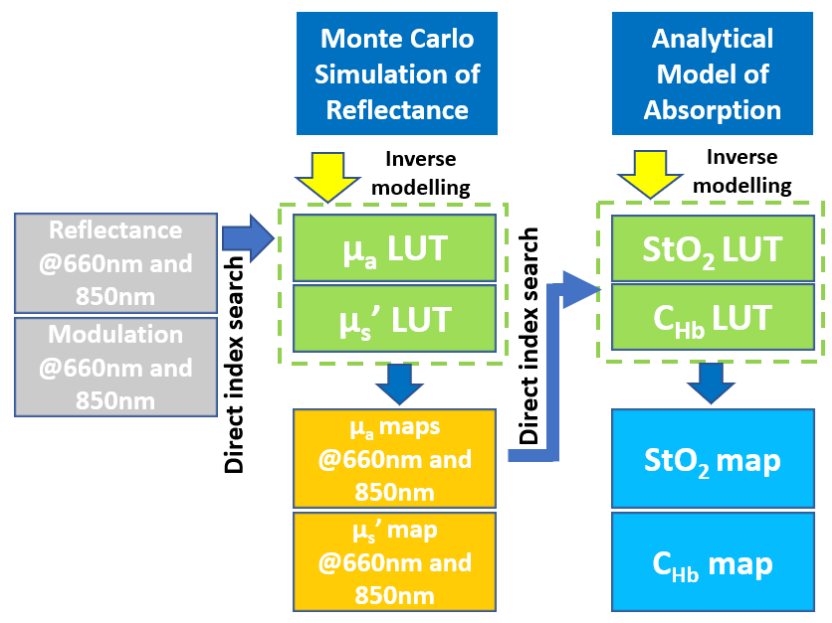

Fig. 1. Overview of two-stage LUT. Monte Carlo simulation of reflectance and analytical model of blood absorption are inverted to generate LUTs for optical properties mapping (stage-1) and perfusion mapping (stage-2), respectively. SFDI measurements at $660 \mathrm{~nm}$ and $850 \mathrm{~nm}$ are used as inputs to quantify absorption and reduced scattering coefficients. The absorption coefficients are later used as search indices to quantify oxygen saturation and total hemoglobin concentration. 
The stage-1 LUT was generated by inverse modeling diffuse reflectance obtained by Monte Carlo simulation in the spatial frequency domain, and the stage-2 LUT was generated by inverse modeling of an analytical model of tissue absorption. Quantification of tissue perfusion properties was based on SFDI imaging at 660nm and $850 \mathrm{~nm}$. These two wavelengths located on the opposite side of the isosbestic wavelength, around $800 \mathrm{~nm}$, have been previously demonstrated to quantify blood perfusion properties effectively [16]. With the two-stage LUT, reflectance and modulation measurements at both $660 \mathrm{~nm}$ and $850 \mathrm{~nm}$ were first used as search indices to determine absorption and reduced scattering coefficient using the stage- 1 LUT. The absorption coefficients at $660 \mathrm{~nm}$ and $850 \mathrm{~nm}$ were subsequently used as searching indices to efficiently determine oxygen saturation and blood concentration using the stage-2 LUT. In the rest of the section, detailed procedures of generating and evaluating the performance of two-stage LUT for quantifying perfusion properties will be presented.

\subsection{Stage-1 LUT for quantifying optical properties}

Monte Carlo (MC) simulation has been widely used for simulating light transport in turbid media. The simulation results are often used to generate LUT tables for quantifying optical properties through inverse modeling $[19,20]$. Traditionally, MC simulation was implemented on CPU [21], and is considered computationally intensive and slow [22]. Due to its highly parallel nature of execution, graphic computing unit (GPU) based MC simulation programs have recently been developed, which significantly improve the simulation efficiency [23]. This study adopted the Monte Carlo eXtreme (MCX) simulation package developed by Fang and Boas to simulate reflectance in the spatial frequency domain [24].

We simulated reflectance images under structured illumination with a spatial frequency of $0.125 \mathrm{~mm}^{-1}$ on large virtual phantoms $(9.6 \mathrm{~cm} \times 9.6 \mathrm{~cm} \times 5 \mathrm{~cm})$ with a grid size of $0.1 \mathrm{~mm}$ in $\mathrm{x}, \mathrm{y}$, and $\mathrm{z}$ directions. The sample thickness of $5 \mathrm{~cm}$ was chosen to satisfy the requirement for infinite geometry in MC simulations [18]. To reflect the general properties of biological tissue, the virtual phantom was assumed to have an anisotropy $\mathrm{g}=0.9$, and a refractive index $\mathrm{n}=1.37$ [25]. A total of 208 pairs of biologically relevant optical properties were simulated with 16 absorption coefficient $\left(\mu_{\mathrm{a}}\right)$ values from $0.001 \mathrm{~mm}^{-1}$ to $1 \mathrm{~mm}^{-1}$, and 13 reduced scattering coefficient values $\left(\mu_{\mathrm{s}}{ }^{\prime}\right)$ from $0.5 \mathrm{~mm}^{-1}$ to $3 \mathrm{~mm}^{-1}$. For each sample, diffuse reflectance images were simulated under 3 phasestepped $\left(0^{\circ}, 120^{\circ}\right.$, and $\left.240^{\circ}\right)$ structured illuminations. Thus, a total of $624 \mathrm{MC}$ simulations were performed, and the corresponding reflectance images were saved. To reduce the noises in simulation, a total of 200 million photons was launched in each simulation.

Following the simulation, a sub-region (red-dash square in Fig. 2A) of each reflectance image was isolated to reduce the signal fall-off toward the edge of the image. The resultant diffuse reflectance image was then averaged along the pattern direction (yellow arrows in Fig. 2A) to further improve the signal quality. Three intensity profiles, $I_{1}, I_{2}$ and $I_{3}$, were obtained for three phase-stepped illuminations (Fig. 2B). The demodulation process, shown in Eq. 1 and 2 was then performed to extract DC $\left(I_{D C}\right)$ and $\mathrm{AC}\left(I_{A C}\right)$ signals (Fig. 2c). 

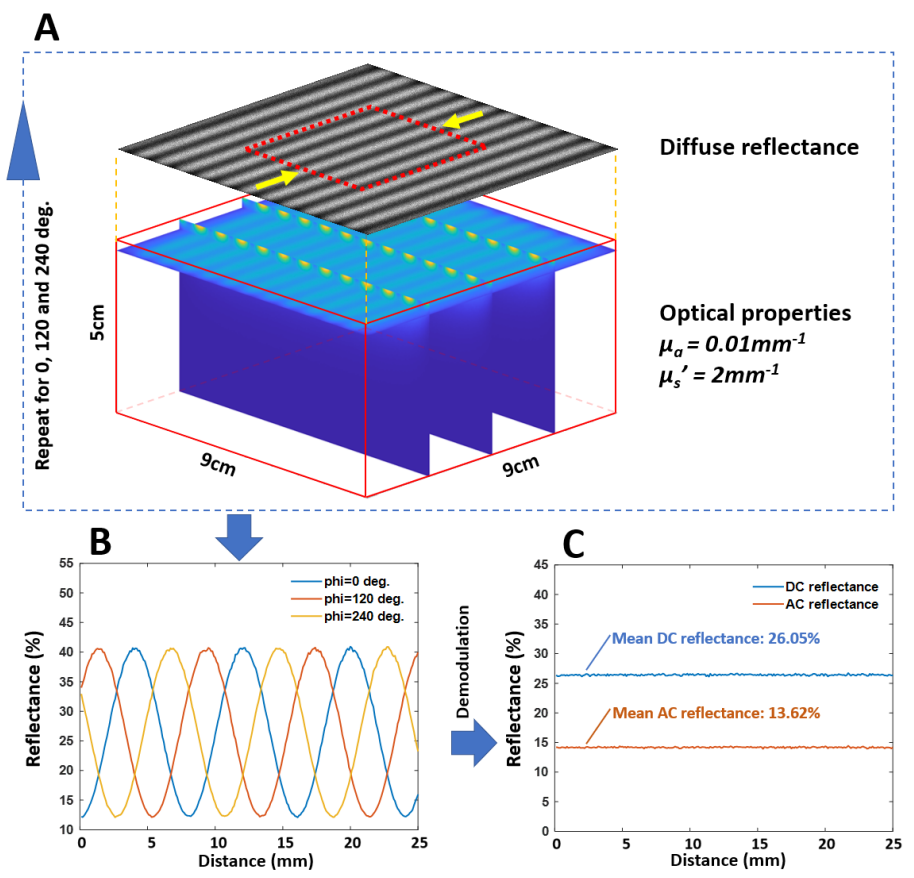

Fig. 2. Monte Carlo simulation workflow. A.Schematic of Monte Carlo simulation over a large volume. For each setting, the simulation was performed with phase shifts of 0,120 , and 240 degrees. The central region (red dash box) of the diffuse reflectance image (gray-scale image) was averaged along the direction of yellow arrows to obtain reflectance plots. B. Reflectance plots at three phase shifts. C. Demodulated $\mathrm{AC}$ and DC intensity plots.

$$
\begin{aligned}
& I_{D C}=1 / 3\left(I_{1}+I_{2}+I_{3}\right) \\
& I_{A C}=\sqrt{2} / 3 \sqrt{\left(I_{1}-I_{2}\right)^{2}+\left(I_{2}-I_{3}\right)^{2}+\left(I_{3}-I_{1}\right)^{2}}
\end{aligned}
$$

$I_{D C}$ and $I_{A C}$ signals were further converted to reflectance (R) and modulation (M) with Eq. 3 and 4, respectively.

$$
\begin{aligned}
& R\left(\mu_{a}, \mu_{s}^{\prime}\right)=I_{D C}\left(\mu_{a}, \mu_{s}^{\prime}\right) \\
& M\left(\mu_{a}, \mu_{s}^{\prime}\right)=\frac{I_{A C}\left(\mu_{a}, \mu_{s}^{\prime}\right)}{I_{D C}\left(\mu_{a}, \mu_{s}^{\prime}\right)}
\end{aligned}
$$

Maps of $R\left(\mu_{a}, \mu_{s}^{\prime}\right)$ and $M\left(\mu_{a}, \mu_{s}^{\prime}\right)$ were converted to LUTs, $\mu_{s}^{\prime}(R, M)$ and $\mu_{s}^{\prime}(R, M)$, using a coordinate mapping technique similar to the one described elsewhere [15]. Briefly, after obtaining $R\left(\mu_{a}, \mu_{s}^{\prime}\right)$ and $M\left(\mu_{a}, \mu_{s}^{\prime}\right)$ from the simulation, a 2D interpolation was performed to generate another set of $\mathrm{R}$ and $\mathrm{M}$ maps with a finer and uniform grid size of $\mu_{\mathrm{a}}$ and $\mu_{\mathrm{s}}$ '. Data points in the newly generated $R\left(\mu_{a}, \mu_{s}^{\prime}\right)$ and $M\left(\mu_{a}, \mu_{s}^{\prime}\right)$ maps were directly mapped to generate two new maps $\mu_{a}(R, M)$ and $\mu_{s}^{\prime}(R, M)$. A scattered data interpolation was used to fill the gaps in $\mu_{a}(R, M)$ and $\mu_{s}^{\prime}(R, M)$ maps. To facilitate a rapid search for optical properties, during the LUT generation, reflectance and 
modulation were up-scaled by a factor of 1000 , and rounded to the nearest integer. This operation allowed for direct index searching based on reflectance measurements.

\subsection{Stage-2 LUT for quantifying perfusion properties}

We performed forward modeling of tissue absorption at two wavelengths, $660 \mathrm{~nm}$ and $850 \mathrm{~nm}$. Hemoglobin concentration, $\mathrm{CHb}$, was varied from $0.075 \mathrm{~g} / \mathrm{L}$ to $22.5 \mathrm{~g} / \mathrm{L}$ with an increment of $0.075 \mathrm{~g} / \mathrm{L}$, which corresponds to $0.05 \%$ and $15 \%$ of the total hemoglobin concentration of $150 \mathrm{~g} / \mathrm{L}$ in the whole blood. This $\mathrm{CHb}$ range reflects the majority types of organs except for the kidney and the liver [25]. $\mathrm{CHb}$ in those two organs is well above $30 \%$ [25]. Oxygen saturation, $\mathrm{StO}_{2}$, was varied from $50 \%$ to $100 \%$ with an increment of $0.25 \%$. A hemoglobin molecular weight of $64500 \mathrm{~g} / \mathrm{mole}$ was used in the simulation [26]. The absorption coefficient at both wavelengths can be analytically described by Eq. 5 and 6, respectively, where $\varepsilon_{\mathrm{HbO}_{2}}$ and $\varepsilon_{\mathrm{Hb}}$ are molar extinction coefficients of oxygenated and deoxygenated hemoglobin. With a similar technique described in the previous section, maps of $\mu_{a_{-6} 660}(\mathrm{StO} 2, \mathrm{CHb})$ and $\mu_{a_{-} 850}(\mathrm{StO} 2, \mathrm{CHb})$ were converted to maps of $\operatorname{StO} 2\left(\mu_{a_{-} 660}, \mu_{a_{-} 850}\right)$ and $\mathrm{CHb}\left(\mu_{a_{-} 660}, \mu_{a_{-} 850}\right)$, and a scaling factor of 5000 was used.

$$
\begin{aligned}
& \left.\mu_{a_{-} 660}(\mathrm{StO} 2, \mathrm{CHb})=\frac{\log (10)}{64500}\left[\varepsilon_{\mathrm{HbO}_{2} 660} \cdot(\mathrm{StO} 2 \cdot \mathrm{CHb})+\varepsilon_{\mathrm{Hb}_{-} 660} \cdot(1-\mathrm{StO} 2) \cdot \mathrm{CHb}\right)\right] \\
& \left.\mu_{a_{-} 850}(\mathrm{StO} 2, \mathrm{CHb})=\frac{\log (10)}{64500}\left[\varepsilon_{\mathrm{HbO}_{2} 850} \cdot(\mathrm{StO} 2 \cdot \mathrm{CHb})+\varepsilon_{H b_{-} 850} \cdot(1-\mathrm{StO} 2) \cdot \mathrm{CHb}\right)\right]
\end{aligned}
$$

\subsection{Quantification speed test}

Speed of quantifying optical and perfusion properties using the two-stage LUT was evaluated based on a set of simulated diffuse reflectance images (Section 2.3.1 and 2.3.2). The performance of stage-2 LUT was compared to the Beer-Lambert Law based quantification (Section 2.3.3). All quantification methods were implemented on both CPU and GPU to fully evaluate their efficiency. GPU acceleration was achieved by simply converting a regular MATLAB array into a GPU-compatible array with the MATLAB function gpuArray ( ). The testing platform consists of an Intel CPU (i9 10900K), 32 GB of memory, and a Nvidia GPU (RTX 3080). A speed test script was written in MATLAB, and only the built-in MATLAB functions were used. We performed a speed test on three sets of simulated images with resolutions $512 \times 512$ pixels, $1024 \times 1024$ pixels, and 2048×2048 pixels. Each set of images consisted of 50 images. Three image sizes were later referred to as small, medium, and large sizes, respectively.

\subsubsection{Optical property mapping using stage-1 LUT}

Reflectance and modulation images obtained from the demodulation were first multiplied by a scaling factor of 1000, and rounded to the nearest integer. MATLAB function sub2ind() was used to convert $2 \mathrm{D}$ maps of scaled reflectance and modulation into linear search indices for stage-1 LUT to quantify optical properties, as shown in Fig. 3A and 3B.

\subsubsection{Perfusion property mapping using stage-2 LUT}


Following optical property quantification, absorption coefficient maps at $660 \mathrm{~nm}$ and $850 \mathrm{~nm}$ were multiplied by a scaling factor of 5000, and similarly, the integer portion of the result was retained. MATLAB function sub2ind () was used to convert 2D maps of scaled absorption coefficient maps into linear search indices for stage-2 LUT to quantify perfusion properties, as shown in Fig. 3C and 3D.

\subsubsection{Perfusion property mapping using Beer-Lambert Law}

Eq. 5 and 6 were solved for concentration of oxygenated hemoglobin $(=\mathrm{StO} 2 \cdot \mathrm{CHb})$ and deoxygenated hemoglobin $(=(1-\mathrm{StO} 2) \cdot \mathrm{CHb})$, based on which oxygen saturation and total hemoglobin concentration were determined. To improve the computation efficiency, 2D absorption maps were first converted to $1 \mathrm{D}$ array to take the advantage of high efficiency of vectorized computation in MATLAB.

\section{4 in-silico validation}

We examined the quantification accuracy of the perfusion parameters through an insilico validation procedure. We generated five sets of digital phantoms with total hemoglobin concentrations varying from $2.25 \mathrm{~g} / \mathrm{L}$ to $20.25 \mathrm{~g} / \mathrm{L}$ with an increment of $4.5 \mathrm{~g} / \mathrm{L}$. At each hemoglobin concentration, five phantoms were created with the oxygen saturation levels varying from $50 \%$ to $100 \%$ with an increment of $10 \%$. Thus, a total of 30 phantoms were generated. Absorption coefficients at $660 \mathrm{~nm}$ and $850 \mathrm{~nm}$ were calculated using Eq. (5) and (6) for all phantoms. The absorption coefficients were later used to quantify hemoglobin concentration and oxygen saturation using the stage-2 LUT. Quantification errors were calculated based on ground truth values.

\section{Results}

\subsection{Two-stage LUT}

Figure 3 exhibits a two-stage LUT for optical and perfusion property mapping. The axes of each LUT are characterized by actual values and their corresponding search indices. In both stages, the corresponding search indices are used to determine the optical and perfusion properties. 


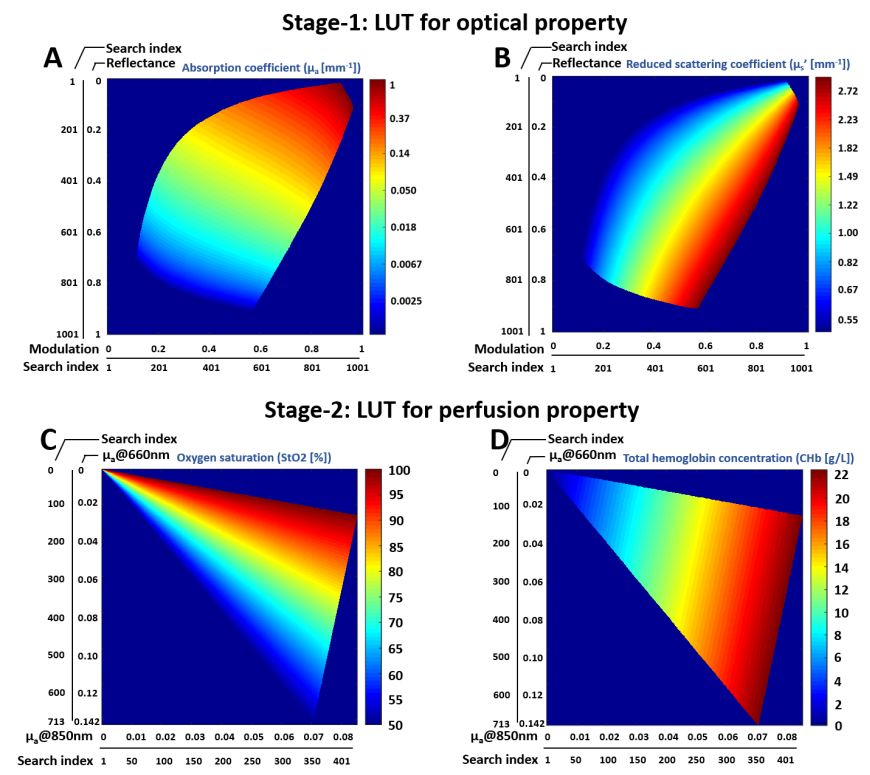

Fig. 3. Two-stage LUTs for stage-1 optical (A and B) and stage-2 perfusion (C and D) properties mapping. Reflectance and modulation in stage-1 LUT, and absorption coefficients at $660 \mathrm{~nm}$ and $850 \mathrm{~nm}$ in stage- 2 LUT were converted into search indices.

\subsection{Quantification speed test}

Table 1 summarizes the processing time for quantifying optical properties and perfusion properties using both GPU- and CPU-based computation. It is clear that with the GPU acceleration, the processing time of three types of quantification have been reduced. Note that the LUT access time (processing time in the parentheses) accounts for a smaller portion of the over processing time in the GPU-based processing than that in CPU-based processing.

Table 1 Processing time for optical and perfusion property quantification with GPU and CPU

\begin{tabular}{|c|c|c|c|c|c|c|c|}
\hline & & \multicolumn{3}{|c|}{ GPU-based } & \multicolumn{3}{|c|}{ CPU-based } \\
\hline \multicolumn{2}{|c|}{ Image size (pixels x pixels) } & $512(\mathrm{~S})$ & 1024(M) & $2048(\mathrm{~L})$ & $512(\mathrm{~S})$ & 1024(M) & 2048(L) \\
\hline \multirow{3}{*}{$\begin{array}{l}\text { Processing } \\
\text { time (ms) }\end{array}$} & $\begin{array}{l}\text { I. } \mu_{a} \text { and } \mu_{s}^{\prime} \text { mapping at } \\
660 \mathrm{~nm} \text { and } 850 \mathrm{~nm} \text { with } \\
\text { stage-1 LUT }\end{array}$ & $\begin{array}{c}2.36 \\
(0.87)^{*}\end{array}$ & $\begin{array}{c}3.39 \\
(1.04)\end{array}$ & $\begin{array}{c}6.94 \\
(2.47)\end{array}$ & $\begin{array}{l}10.64 \\
(8.19)\end{array}$ & $\begin{array}{c}41.72 \\
(31.05)\end{array}$ & $\begin{array}{c}170.34 \\
(128.84)\end{array}$ \\
\hline & $\begin{array}{l}\text { II. St } \mathrm{O}_{2} \text { and } \mathrm{CHb} \text { mapping } \\
\text { with stage-2 LUT }\end{array}$ & $\begin{array}{c}1.41 \\
(0.19)\end{array}$ & $\begin{array}{c}2.34 \\
(0.22)\end{array}$ & $\begin{array}{c}6.49 \\
(0.36)\end{array}$ & $\begin{array}{c}4.87 \\
(2.69)\end{array}$ & $\begin{array}{c}20.52 \\
(10.84)\end{array}$ & $\begin{array}{c}86.61 \\
(45.96)\end{array}$ \\
\hline & $\begin{array}{l}\text { III. St } \mathrm{O}_{2} \text { and } \mathrm{CHb} \text { mapping } \\
\text { using Beer-Lambert Law }\end{array}$ & 9.18 & 24.72 & 93.33 & 9.48 & 37.91 & 165.81 \\
\hline
\end{tabular}

${ }^{*}$ GPU access time.

While the quantifications for the perfusion property with both stage-2 LUT and BeerLambert law were accelerated with a GPU, stage-2 LUT clearly showed a much higher efficiency. The stage-2 LUT is approximately 5.5 times and 13.5 times faster for small and large sized images, respectively. Without GPU acceleration, stage-2 LUT still showed about $90 \%$ higher efficiency than the Beer-Lambert Law method.

It is worth noting that a large image size imposes a much higher penalty on CPU-based processing than GPU-based processing. The CPU-based processing time is 
proportional $(1 \mathrm{x}, 4 \mathrm{x}$, and $16 \mathrm{x})$ to the image sizes for all three types of quantifications. On the other hand, the GPU-based processing time is less affected by the image sizes, especially for the LUT-based quantifications

Converting the processing time to processing speed (frames per second/FPS) revealed GPU-based two-stage LUT (sum of processing time I and II) was capable of achieving a processing speed of 266, 174, and 74 FPS for small, median, and large sized images, respectively, which is 2.1, 3.9, and 6.5 times faster than that of the Beer-Lambert Law based processing (sum of processing time I and III) (Fig. 4A). On the other hand, CPUbased quantification was slower, but it still achieved approximately 65,16 , and 4 FPS with two-stage LUT for three image sizes, which is about $30 \%$ faster than BeerLambert law-based processing (Fig. 4B). Note that the processing time of optical properties includes both absorption and reduced scattering coefficients. If tissue scattering property is not of interest, excluding scattering quantification could further improve the quantification speed.

A

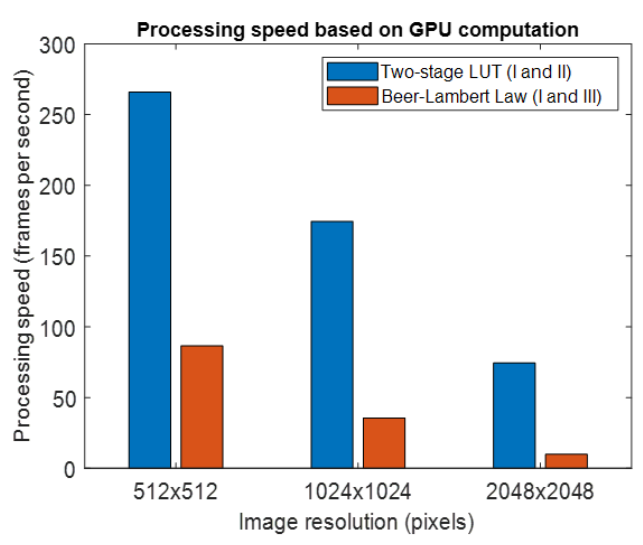

B

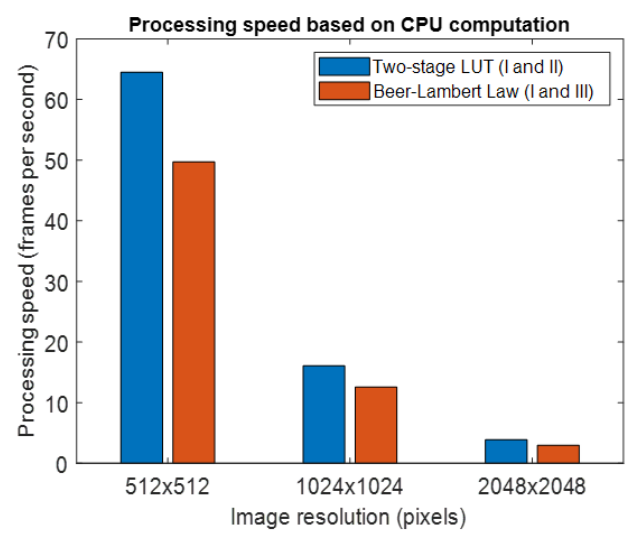

Fig. 4. A. Quantification frame rate of optical and perfusion properties of three image sizes with a GPU and a CPU. B. Processing time increases as the image size increases for both GPU- and CPU- based processing. However, the GPU-based is less affected.

\section{3 in-silico validation}

The stage-2 LUT exhibited a high accuracy of quantifying perfusion properties as shown in Fig.5. The absolute quantification error is less than 3.5\% for total hemoglobin concentration and less than $2.5 \%$ for oxygen saturation over a wide range of perfusion properties. While the overall error is small, Fig. 5 suggests that a lower total hemoglobin concentration may correlate with a higher error. 
A

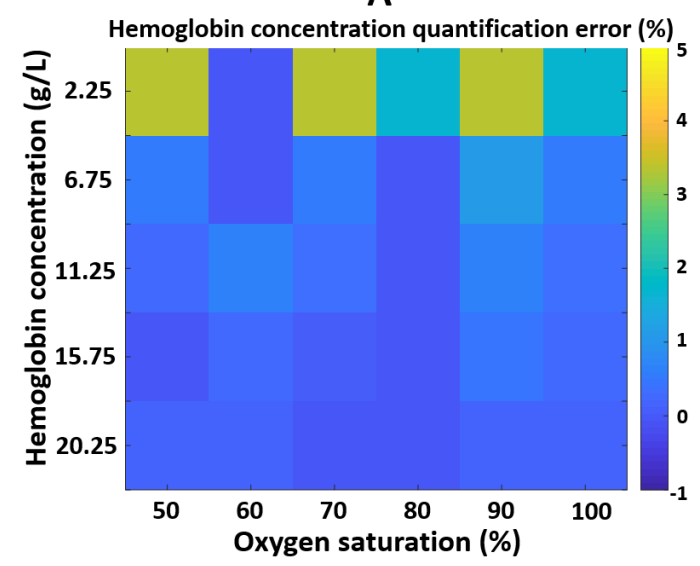

B

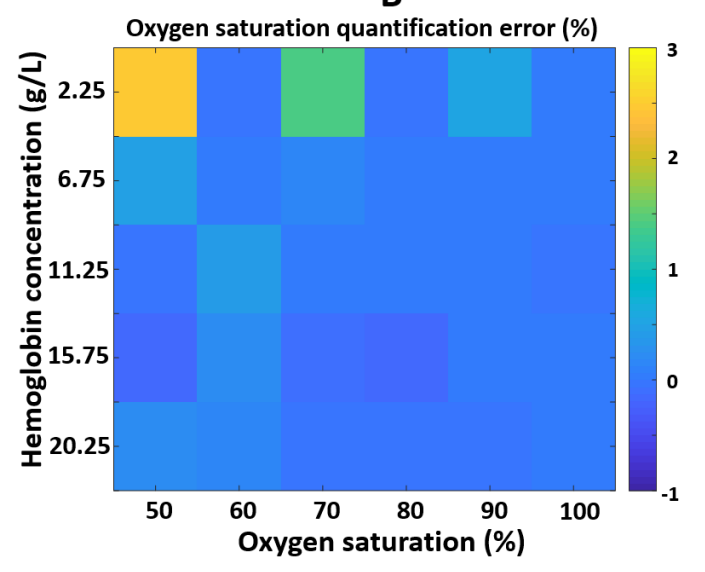

Fig. 5. Quantification accuracy analysis of hemoglobin concentration and oxygen saturation with stage-2 LUT.

\section{Discussion}

In this study, we developed and demonstrated a highly efficient and accurate two-stage LUT method that quantifies the tissue's optical and perfusion properties with SFDI. High efficiency was achieved through direct index searching of two LUTs, which eliminated time-consuming fitting operations to determine optical and subsequently perfusion properties. GPU-based implementation significantly improved the searching speed through parallelization. At an image size of $2048 \times 2048$ pixels, we achieved a quantification speed of 74 FPS for both optical and perfusion properties.

In recent years, methods for direct quantification of optical and perfusion properties using artificial intelligence have been reported to improve the processing speed towards real-time applications. Zhao et al. reported a processing time of $94.4 \mathrm{~ms}$ (excluding time for image import and result display) for quantifying perfusion properties for images with a size of $540 \times 720$ pixels using a deep residual network [17]. Using adversarial deep learning, Chen and Durr reported that approximately 40ms was need to infer optical and perfusion properties for images with a size of $512 \times 512$ pixels [16]. It is worth noting that both studies utilized two wavelengths at around $660 \mathrm{~nm}$ and $850 \mathrm{~nm}$, which are the same as the wavelengths used in this study.

A two-stage LUT method is highly suitable for real-time applications. With GPU acceleration, it achieved 266 FPS and 74 FPS for small and large sized images. The short processing time could provide ample room to accommodate more sophisticated algorithms while still maintaining real-time performance. When the two-stage LUT is coupled with a dual-sensor SFDI imaging system, reflectance images at $660 \mathrm{~nm}$ and $850 \mathrm{~nm}$ could be acquired simultaneously and processed rapidly, which would enable time-sensitive imaging applications, such as real-time tissue viability monitoring.

Two-stage LUTs outperformed the Beer-Lamber Law based quantification regardless of the computational platform. Even with GPU acceleration, Beer-Lamber Law method only achieved 35.5 FPS for median size images. Any additional processing would slow it down to sub-30 FPS, which is not ideal for real-time applications. 
While CPU-based implementation of two-stage LUT is not as fast, it does not require a dedicated GPU and offer a few advantages. First, the two-stage LUT is still suitable for offline applications where processing time is not a critical factor, and it is about $30 \%$ more efficient than Beer-Lamber Law approach. Second, the two-stage LUT offered approximately a processing speed of 65 FPS for small sized images, which still could enable real-time applications when the image resolution is not a concern. It is worth noting that in real life applications, acquiring and transferring images to memory, and displaying the processed results take additional time. To preserve the high performance of two-stage LUT, multi-threaded architecture could be adopted. Each thread runs independently and is only responsible for its own task, such as image transfer, quantification, or result display.

The two-stage LUT quantification method presented in this study was intended to serve as a general framework to map the optical and perfusion properties of the tissue. Implementing this general framework requires additional considerations. Firstly, the optical property LUT was generated using MC simulation on homogenous phantoms. While MC simulation is highly accurate, the LUT is not imaging system-specific. When applying this LUT to quantify optical properties of the sample imaged with a specific SFDI system, additional calibration and scaling of the LUT are likely needed to match the characteristics of the imaging system and to achieve a high quantification accuracy. Secondly, the sample's 3D surface shape affects the diffuse reflectance, and thus the quantification, which was not accounted for in our method. Multiple techniques have been reported to correct for surface height, and these methods could be adopted to improve quantification accuracy $[13,27]$. Finally, the two-stage LUT was based on two wavelengths that are sensitive to oxygenated and deoxygenated hemoglobin. If the tissue to be measured presents additional major chromophores, such as melanin, this method may not yield a high quantification accuracy. Higherdimensional (e.g., three dimensional) LUT could be generated to account for additional chromophores.

\section{Conclusion}

In this work, we presented a highly efficient method, two-stage LUT, that quantifies the tissue's optical and perfusion properties in SFDI imaging. Both GPU- and CPUbased implementations demonstrated a high efficiency. This method can potentially be adapted and incorporated into existing SFDI imaging applications to improve data processing efficiency. In future work, we will implement this two-stage LUT in a dualwavelength SFDI imaging system to monitor tissue perfusion and hemodynamics in real-time.

Acknowledgments. This research work was supported by the Department of Engineering and Faculty Startup Funds provided by the Provost Office at Duquesne University.

Disclosures. The author declares no conflict of interest.

Data availability. The data and code that support the findings of this study are available from the corresponding author upon reasonable request. 


\section{References}

1. O. Ndubuizu, and J. C. LaManna, "Brain tissue oxygen concentration measurements," Antioxidants \& redox signaling 9, 1207-1220 (2007).

$2 . \quad$ R. Boushel, H. Langberg, J. Olesen, J. Gonzales-Alonzo, J. Bülow, and M. Kjaer, "Monitoring tissue oxygen availability with near infrared spectroscopy (NIRS) in health and disease," Scandinavian journal of medicine \& science in sports 11, 213-222 (2001).

3. N. Rajaram, A. F. Reesor, C. S. Mulvey, A. E. Frees, and N. Ramanujam, "Noninvasive, simultaneous quantification of vascular oxygenation and glucose uptake in tissue," PloS one 10, e0117132 (2015).

4. K. J. Zuzak, M. D. Schaeberle, E. N. Lewis, and I. W. Levin, "Visible reflectance hyperspectral imaging: characterization of a noninvasive, in vivo system for determining tissue perfusion," Analytical chemistry 74, 2021-2028 (2002).

5. D. J. Cuccia, F. Bevilacqua, A. J. Durkin, and B. J. Tromberg, "Modulated imaging: quantitative analysis and tomography of turbid media in the spatial-frequency domain," Optics letters 30, 1354-1356 (2005).

6. D. J. Cuccia, F. P. Bevilacqua, A. J. Durkin, F. R. Ayers, and B. J. Tromberg, "Quantitation and mapping of tissue optical properties using modulated imaging," Journal of biomedical optics 14, 024012 (2009).

7. S. Gioux, A. Mazhar, and D. J. Cuccia, "Spatial frequency domain imaging in 2019: principles, applications, and perspectives," Journal of biomedical optics 24, 071613 (2019).

8. J. R. Weber, A. J. Durkin, B. J. Tromberg, D. J. Cuccia, W. R. Johnson, D. W. Wilson, G. H. Bearman, M. Hsu, A. Lin, and D. K. Binder, "Multispectral imaging of tissue absorption and scattering using spatial frequency domain imaging and a computedtomography imaging spectrometer," Journal of biomedical optics 16, 011015 (2011). 9. A. Mazhar, S. Dell, D. J. Cuccia, S. Gioux, A. J. Durkin, J. V. Frangioni, and B. J. Tromberg, "Wavelength optimization for rapid chromophore mapping using spatial frequency domain imaging," Journal of biomedical optics 15, 061716 (2010).

10. M. Erfanzadeh, S. Nandy, P. D. Kumavor, and Q. Zhu, "Low-cost compact multispectral spatial frequency domain imaging prototype for tissue characterization," Biomedical optics express 9, 5503-5510 (2018).

11. R. H. Wilson, K. P. Nadeau, F. B. Jaworski, R. Rowland, J. Q. M. Nguyen, C. Crouzet, R. B. Saager, B. Choi, B. J. Tromberg, and A. J. Durkin, "Quantitative short-wave infrared multispectral imaging of in vivo tissue optical properties," Journal of biomedical optics 19, 086011 (2014).

12. J. Vervandier, and S. Gioux, "Single snapshot imaging of optical properties," Biomedical optics express 4, 2938-2944 (2013).

13. M. van de Giessen, J. P. Angelo, and S. Gioux, "Real-time, profile-corrected single snapshot imaging of optical properties," Biomedical optics express 6, 4051-4062 (2015).

14. E. Aguénounon, J. T. Smith, M. Al-Taher, M. Diana, X. Intes, and S. Gioux, "Realtime, wide-field and high-quality single snapshot imaging of optical properties with profile correction using deep learning," Biomedical Optics Express 11, 5701-5716 (2020).

15. J. Angelo, C. R. Vargas, B. T. Lee, I. J. Bigio, and S. Gioux, "Ultrafast optical property map generation using lookup tables," Journal of biomedical optics 21, 110501 (2016).

16. M. T. Chen, and N. J. Durr, "Rapid tissue oxygenation mapping from snapshot structured-light images with adversarial deep learning," Journal of Biomedical Optics 25, 112907 (2020).

17. Y. Zhao, Y. Deng, S. Yue, M. Wang, B. Song, and Y. Fan, "Direct mapping from diffuse reflectance to chromophore concentrations in multi-fx spatial frequency domain imaging (SFDI) with a deep residual network (DRN)," Biomedical Optics Express 12, 433$443(2021)$. 
18. T. A. Erickson, A. Mazhar, D. J. Cuccia, A. J. Durkin, and J. W. Tunnell, "Lookuptable method for imaging optical properties with structured illumination beyond the diffusion theory regime," Journal of biomedical optics 15, 036013 (2010).

19. R. J. Hennessy, S. L. Lim, M. K. Markey, and J. W. Tunnell, "Monte Carlo lookup table-based inverse model for extracting optical properties from tissue-simulating phantoms using diffuse reflectance spectroscopy," Journal of biomedical optics 18, 037003 (2013).

20. X. Zhong, X. Wen, and D. Zhu, "Lookup-table-based inverse model for human skin reflectance spectroscopy: two-layered Monte Carlo simulations and experiments," Optics express 22, 1852-1864 (2014).

21. D. Marti, R. N. Aasbjerg, P. E. Andersen, and A. K. Hansen, "MCmatlab: an opensource, user-friendly, MATLAB-integrated three-dimensional Monte Carlo light transport solver with heat diffusion and tissue damage," Journal of biomedical optics 23, 121622 (2018).

22. L. Wang, S. L. Jacques, and L. Zheng, "MCML-Monte Carlo modeling of light transport in multi-layered tissues," Computer methods and programs in biomedicine 47, 131146 (1995).

23. N. Ren, J. Liang, X. Qu, J. Li, B. Lu, and J. Tian, "GPU-based Monte Carlo simulation for light propagation in complex heterogeneous tissues," Optics express 18, 68116823 (2010).

24. Q. Fang, and D. A. Boas, "Monte Carlo simulation of photon migration in 3D turbid media accelerated by graphics processing units," Optics express 17, 20178-20190 (2009).

25. S. L. Jacques, "Optical properties of biological tissues: a review," Physics in Medicine \& Biology 58, R37 (2013).

26. V. L. Clark, and J. A. Kruse, "Clinical methods: the history, physical, and laboratory examinations," Jama 264, 2808-2809 (1990).

27. S. Gioux, A. Mazhar, D. J. Cuccia, A. J. Durkin, B. J. Tromberg, and J. V. Frangioni, "Three-dimensional surface profile intensity correction for spatially modulated imaging," Journal of biomedical optics 14, 034045 (2009). 